\title{
Transcriptional regulation of kinases downstream of the T cell receptor: another immunomodulatory mechanism of glucocorticoids
}

Maria Grazia Petrillo ${ }^{1 \dagger}$, Katia Fettucciari ${ }^{2 \dagger}$, Paolo Montuschi ${ }^{3 \dagger}$, Simona Ronchetti ${ }^{1}$, Luigi Cari ${ }^{1}$, Graziella Migliorati ${ }^{1}$, Emanuela Mazzon ${ }^{4}$, Oxana Bereshchenko ${ }^{1}$, Stefano Bruscoli ${ }^{1}$, Giuseppe Nocentini ${ }^{1,5^{*}}$ and Carlo Riccardi ${ }^{1}$

\begin{abstract}
Background: Glucocorticoids affect peripheral immune responses, including modulation of T-cell activation, differentiation, and apoptosis. The quantity and quality of T-cell receptor (TCR)-triggered intracellular signals modulate T-cell function. Thus, glucocorticoids may affect T cells by interfering with the TCR signaling cascade. The purpose of the study was to search for glucocorticoid-modulated kinases downstream of the TCR.

Methods: Gene modulation in lymphoid cells either treated with glucocorticoids or from glucocorticoid-treated mice was studied using a RNase protection assay, real-time PCR, and western blotting. The sensitivity of genetically modified thymocytes to glucocorticoid-induced apoptosis was studied by performing hypotonic propidium iodide staining and flow cytometry. The Student's t-test was employed for statistical evaluation.

Results: We found that transcription of Itk, a non-receptor tyrosine kinase of the Tec family, was up-regulated in a mouse T-cell hybridoma by the synthetic glucocorticoid dexamethasone. In contrast, dexamethasone down-regulated the expression of Txk, a Tec kinase that functions redundantly with Itk, and Lck, the Src kinase immediately downstream of the TCR. We investigated the expression of Itk, Txk, and Lck in thymocytes and mature lymphocytes following in vitro and in vivo dexamethasone treatment at different time points and doses. Kinase expression was differentially modulated and followed distinct kinetics. Itk was up-regulated in all cell types and conditions tested. Txk was strongly up-regulated in mature lymphocytes but only weakly up-regulated or non-modulated in thymocytes in vitro or in vivo, respectively. Conversely, Lck was down-regulated in thymocytes, but not modulated or up-regulated in mature lymphocytes in the different experimental conditions. This complex behaviour correlates with the presence of both positive and negative glucocorticoid responsive elements (GRE and nGRE, respectively) in the Itk, Txk and Lck genes. To investigate the function associated with Itk up-regulation, dexamethasone-induced apoptosis of thymocytes from Itk-deficient mice was evaluated. Our results demonstrated that Itk deficiency causes increased sensitivity to dexamethasone but not to other pro-apoptotic stimuli.
\end{abstract}

Conclusions: Modulation of Itk, Txk, and Lck in thymocytes and mature lymphocytes is another mechanism by which glucocorticoids modulate T-cell activation and differentiation. Itk up-regulation plays a protective role in dexamethasone-treated thymocytes.

Keywords: Glucocorticoids, Gene modulation, Kinases, In vivo treatment, T cells, Thymocytes, Apoptosis, Knock-out mice

\footnotetext{
* Correspondence: giuseppe.nocentini@unipg.it

${ }^{+}$Equal contributors

'Department of Medicine, University of Perugia, Perugia, Italy

${ }^{5}$ Department of Medicine, Section of Pharmacology, Severi Square 1,

University of Perugia, I-06132 San Sisto, Perugia, Italy

Full list of author information is available at the end of the article
} 


\section{Background}

Glucocorticoids are used to treat several autoimmune diseases and prevent organ rejection following transplantation due to their potent anti-inflammatory and immunosuppressive activity. These compounds can also inhibit lymphocyte proliferation and induce lymphocyte death [1-3]. However, glucocorticoids have also been reported to potentiate the immune response and modulate lymphocyte differentiation. For example, when used in some experimental systems, these compounds stimulate $\mathrm{T}$-cell receptor (TCR)-mediated T-cell proliferation and inhibit activation-induced cell death [4]. At physiologic concentrations, glucocorticoids shift immunity from a $\mathrm{T}$ helper (Th) 1 to a Th2 response, and promote production of $\mathrm{T}$ regulatory (Treg) cells [5-7]. This shift is also observed following long-term treatment with glucocorticoids [8].

Recognition of the antigen-major histocompatibility complex $(\mathrm{MHC})$ by the TCR leads to a cascade of signaling events initiated by activation of lymphocyte protein tyrosine kinase (Lck), a Src-family kinase crucial in T-cell development and activation [9]. Lck phosphorylates the cytoplasmic domain of CD3, leading to activation of ZAP-70, LAT, and SLP-76, which in turn serve as a platform for recruiting molecules, such as Tec kinases, into the signalosome [10]. Among Tec kinases, IL-2 inducible T cell kinase (Itk) is expressed most highly and exerts the greatest effects on $\mathrm{T}$ cell function $[11,12]$. Txk tyrosine kinase (Txk) is a Tec kinase that exhibits partial redundancy with Itk and is expressed at lower levels than Itk [12]. Mice deficient in Itk $\left(I t k^{-/-}\right)$show altered T-cell development and impaired Tcell effector function. Deletion of both $I t k$ and Txk causes marked defects in TCR responses, including proliferation, cytokine production, and apoptosis in vitro, as well as a dysfunctional immune response to Toxoplasma gondii infection in vivo [13]. Molecular events immediately downstream of the TCR are intact in $T x k^{-/} I t k^{-/-}$cells; however, intermediate events such as inositol triphosphate production, calcium mobilization, and mitogen-activated protein kinase activation are impaired. These data establish Tec kinases as critical regulators of TCR signaling required for phospholipase $\mathrm{C}-\gamma$ activation [13].

Other data suggest that Itk and Txk play a divergent role in $\mathrm{T}$ cell differentiation. $I t k^{-/-}$mice are unable to mount a Th2 response in models of allergic asthma [14], as well as following infection with Leishmania major, Nippostrongylus brasiliensis, and Schistosoma Mansoni, which lead to Th1 cytokine production $[15,16]$. Surprisingly $I t k^{-/-} T x k^{-/-}$mice mount Th2 responses, which possibly suggests that these Tec kinases are involved in Th1/Th2 polarization. Indeed, Txk over-expression has been found to increase IFN- $\gamma$ production. Moreover, increased Txk expression has been observed in patients with Behcet's disease, a disorder associated with increased inflammation and Th1 cytokine production [17].
In this study, we demonstrate that the synthetic glucocorticoid dexamethasone modulates the expression of $L c k, I t k$, and Txk in thymocytes and mature lymphocytes in vitro and in vivo. In addition, we report that Itk upregulation in thymocytes has a functional role, as demonstrated by the increased sensitivity of $I t k^{-/-}$thymocytes to dexamethasone-induced apoptosis. Thus, our data suggest that glucocorticoids modulate T-cell function and the immune response by fine-tuning the expression of Lck and Tec kinases.

\section{Methods}

\section{Animals}

$\mathrm{C} 3 \mathrm{H}$ and $\mathrm{BALB} / \mathrm{c}$ mice were purchased from Jackson Laboratory (Maine, USA). Itk ${ }^{-/-} \mathrm{BALB} / \mathrm{c}$ mice were a kind gift of dr. Locksley [15]. The animals were housed in a controlled environment, provided with standard rodent chow and water ad libitum and kept under specific pathogen-free conditions. Animal care was in compliance with regulation in Italy (Decreto Ministeriale 116192), Europe (Official Journal of European Contract Law 358/1 12/18/1986) and USA (Animal Welfare Assurance No A5594-01, Department of Health and Human Services, USA). The study was approved by the Italian Ministero della Salute.

For in vivo experiments, female mice were injected with $0.2 \mathrm{ml}$ of saline solution or $5 / 25 \mathrm{mg} / \mathrm{Kg}$ dexamethasone in $0.2 \mathrm{ml}$ saline solution and sacrificed 3 or $6 \mathrm{~h}$ after treatment.

\section{Cell isolation and treatment}

Spontaneously dividing $\mathrm{CD}^{+}, \mathrm{CD}^{+}, \mathrm{CD} 2^{+}, \mathrm{CD}_{4} 4^{+}$cells of the OVA-specific hybridoma T-cell line 3DO [18] obtained by recloning the original line in our laboratory were used. Cells were maintained in logarithmic growth at $37^{\circ} \mathrm{C}, 5 \% \mathrm{CO}_{2}$ in RPMI 1640 medium supplemented with fetal bovine serum (10\%, FBS), $10 \mathrm{mM}$ Hepes and antibiotics and were treated with $10^{-7} \mathrm{M}$ dexametasone for $24 \mathrm{~h}$.

Thymuses, spleens and cervical, brachial, axillary, superficial inguinal and mesenteric lymph nodes were isolated from 4- to 5-week old female $\mathrm{C} 3 \mathrm{H}$ (in vitro/vivo gene modulation experiments) or female BALB/c (apoptosis experiments) mice, teased in RPMI 1640 medium and directly processed (in vivo experiment) or resuspended in RPMI 1640 supplemented with fetal bovine serum (10\%, FBS), $10 \mathrm{mM}$ Hepes and antibiotics (in vitro experiments). In treated groups, dexamethasone was added for 1 and $3 \mathrm{~h}$ (RPA experiments and real-time PCR, $10^{-7} \mathrm{M}$ ) or $18 \mathrm{~h}$ (apoptosis experiments, concentration $2.5 \times 10^{-8}-10^{-7} \mathrm{M}$ ).

Total, $\mathrm{CD}^{+}{ }^{+}$and $\mathrm{CD}^{+} \mathrm{T}$ lymphocytes were obtained from spleen. Briefly, after red blood cell lysis, single cell suspension was incubated either with CD4 plus CD8 
microbeads (Miltenyi Biotec, Bergisch Gladbach, Germany) to get $\mathrm{T}$ lymphocytes or separately to obtain $\mathrm{CD}^{+}$and $\mathrm{CD}^{+}$cells. Magnetic retained cells where then eluted from LS columns according to the manufacturer's instructions. The purified cells were shown to be $>98 \% \mathrm{CD}^{+}, \mathrm{CD}^{+}$or $\mathrm{CD}^{+}$cells by flow cytometry.

\section{Promoter analysis}

Putative glucocorticoid responsive element (GRE) and negative GRE (nGRE) sites within the murine regulatory regions of Itk, Txk and Lck genes were analyzed by MatInspector software algorithms (Genomatix). The core similarity was set to $75 \%$, while matrix similarity was set to $\geq 0.85$ (a perfect match to the matrix gets a score of 1).

\section{Differential display and cloning}

RNA from untreated and dexamethasone-treated cells was isolated using Trizol LS reagent (Ambion, Life Technologies) according to the manufacturer's instructions. Briefly, $750 \mu \mathrm{l}$ TRIzol LS was added to $250 \mu \mathrm{l}$ medium containing $20-40 \times 10^{6}$ cells. After centrifugation with chloroform, RNA was precipitated by isopropanol and then washed with 75\% ethanol.

DNA-free RNA $(0.1 \mu \mathrm{g})$ was retrotranscribed (Moloney murine leukemia virus reverse transcriptase from Invitrogen, Life Technologies) with an anchored primer $\left(\mathrm{T}_{11} \mathrm{AC}\right)$ and forty cycles of PCR were performed by using $\mathrm{T}_{11} \mathrm{AC}$ and the OPA 5'-CGCGGAGGTG-3' [19]. Three independent samples of untreated 3DO cells were compared to three samples of $24 \mathrm{~h}$ dexamethasonetreated 3DO cells, by SDS-polyacrylamide gel. The radioactive band present in treated samples yet absent in the untreated cells was extracted, cloned and sequenced.

\section{PCR and RACE}

RNA was purified and retrotranscribed as above specified but poly- $\mathrm{T}$ primer was used. To exclude any contamination from cellular DNA, DNAse-treated RNA was also used. Itk intron 5 was amplified by PCR using the primers 5'-TGGGCTGTGTCTATTCCCTGCCATG-3' and 5'-TAGAATTGTGGAGCTGAACAG-3' and using cDNA from RNA or DNA-free RNA. In order to further exclude that Itk intron was amplified from DNA, PCR was also performed with RNA that was not retrotranscribed. 5' rapid amplification of cDNA ends (RACE) was performed as previously described [20].

\section{RNAse protection assay (RPA)}

RNA was isolated as above specified and RPA was performed as previously specified [21]. Probes for RNase protection were constructed by RT-PCR using primers 5'GCTTGGTGCATATCCTTCATG-3' and 5'-CGGTCAT TTCAGGAACCTGAAG-3' for Itk, 5'AAAACATTCCCA
GCGTCAGAGG-3' and 5'-GCAGCGGCTTGCGCTTC GAAGG-3' for Txk and 5'-GAGCAGAGCGGTGAGTGG TGG-3' and 5'-TGCCGCTCGGCGTCCTTACGG-3' for Lck and inserted in the pCRII vector (Invitrogen, Life Technologies). pCRII-Itk, pCRII-Txk and pCRII-Lck RNA probes were 301, 215, $273 \mathrm{bp}$ long and protected a fragment of 174 (encompassing exon 6 and 7), 88 (encompassing exon 3 and 4), 146 bp (encompassing exon 4-6), respectively. Plasmids were linearized with BamHI (New England Biolabs). The probe giving a 250-bp fragment ( $\beta$-actin probe) protecting $\beta$-actin was purchased from Ambion (Life Technologies), linearized with XbaI, and used as internal control. All the probes were transcribed with T7 RNA polymerase (Ambion, Life Technologies) in the presence of $5 \mu \mathrm{Ci}\left[\alpha{ }^{32} \mathrm{P}\right] \mathrm{UTP} ; \beta$-actin was transcribed in the presence of $0.5 \mu \mathrm{Ci}\left[\alpha-{ }^{32} \mathrm{P}\right] \mathrm{UTP}$. After washing out unincorporated nucleotides by quick spin columns (Fine Sephadex G-50, GE Healthcare, Life Sciences), $5 \times 10^{5} \mathrm{cpm}$ probe $\left(5 \times 10^{4} \mathrm{cpm} \beta\right.$-actin) was hybridized to total cell RNA $(10 \mu \mathrm{g})$ and then incubated overnight at $60^{\circ} \mathrm{C}$.

RNase digestion was performed by using an RNase A (Roche) $(40 \mu \mathrm{g} / \mathrm{ml})$ and an RNase T1 (Ambion, Life Technologies $)(1.5 \mathrm{U} / \mu \mathrm{l})$ solution at $20^{\circ} \mathrm{C}$ for $5 \mathrm{~min}$. The undigested products were treated with phenol-chloroform, precipitated with ethanol, and loaded on a denaturing polyacrylamide sequencing gel. The gel was exposed to Biomax MR Film Kodak (Sigma-Aldrich) with intensifying screens at $-70^{\circ} \mathrm{C}$ for $12 \mathrm{~h}$ to 2 days to obtain images of good quality.

Quantitation of protected bands was obtained evaluating cpm by Imager-Packard [22]. The ratio cpm of Itk, Txk or Lck protected fragment/cpm of $\beta$-actin protected fragment was calculated for each RNA and modulation of gene expression was equal to the ratio "value dexamethasonetreated cells"/"value medium-treated cells" or "value cells from dexamethasone-treated animals"/"value from saline solution-treated animals" or "value from untreated animals", as specified. In vitro and in vivo experiments were performed three times. Cells of in vivo experiments were pooled from 3-4 animals of the same experimental group.

\section{Real time PCR}

RNA was purified as above specified. Conversion of total RNA $(1 \mu \mathrm{g})$ to cDNA was performed with QuantiTect Reverse Transcription protocol (Qiagen).

Real time PCR was done with a 7300 Real time PCR system (Applied Biosystem) real time cycler using specific FAM/MGB dye-labeled TaqMan probes: Itk (Mm 00439862_m1), Txk (Mm 01213032_m1), Lck (Mm 00802897_m1). Gene expression was quantitated relatively to the expression of endogenous control mouse beta-actin (4352341e) VIC/MGB probe amplified in the same tube of investigated genes. All probes were purchased from Applied Biosystem. All experiments were 
carried out in triplicate and the $\Delta \Delta^{\mathrm{Ct}}$ method was used to determine expression of the genes of interest, as previously described [23].

\section{Western blotting}

Cells were washed and lysed in SDS sample buffer for $30 \mathrm{~min}$ on ice. After centrifuging, the cleared lysates were boiled and run in a 10\% SDS-polyacrylamide gel. The proteins were then transferred onto nitrocellulose membranes, which were hybridized with rabbit anti-Itk (Upstate, Merck-Millipore) antibody (Ab). Immunoreactive protein bands were visualized using horseradish peroxidase-conjugated goat anti-rabbit IgG (Pierce, Thermo Scientific) followed by enhanced chemioluminescence (Merck-Millipore).

Western blot plates were scanned and band signal intensities were determined using ImageJ software. Expression levels were normalized to $\beta$-tubulin (Sigma-Aldrich) expression.

\section{Apoptosis}

Cells were treated with $10^{-7} \mathrm{M}$ dexamethasone and plastic-coated anti-CD3 Ab $(0.5 \mu \mathrm{g} / \mathrm{ml})$ (Pharmingen) [24]. To evaluate heath shock-induced apoptosis, cells were kept at $43^{\circ} \mathrm{C}$ for 10,20 or 30 min. Before evaluating the apoptosis levels by ipothonic propidium iodide staining [25], cells were cultured at $37^{\circ} \mathrm{C}, 5 \% \mathrm{CO}_{2}$ for $18 \mathrm{~h}$. Flow cytometric analysis was conducted on a Beckman Coulter EPICS XL-MCL running EXPO32 ADC analysis software.

\section{Statistical and mathematical analysis}

Results were normally distributed and are the mean \pm SD. Student's t-test was adopted for statistical evaluation $\left({ }^{*} \mathrm{P}<0.05,{ }^{* *} \mathrm{P}<0.01,{ }^{* * *} \mathrm{P}<0.001\right)$. In apoptosis experiments, apoptosis caused by the treatment (specific apoptosis percentage) was calculated as follows: $100 \times[\%$ apoptosis (treated cells)-\% apoptosis (medium treated cells)]/[100-\% apoptosis (medium treated cells)].

\section{Results}

Dexamethasone up-regulates Itk expression but downregulates $T x k$ and $L c k$ in $3 D O$ cells

We performed differential display to investigate Tec kinase gene expression in 3DO $\mathrm{T}$ cells that were untreated or treated for $24 \mathrm{~h}$ with the synthetic glucocorticoid dexamethasone. A 275-bp cDNA segment was amplified at much higher levels in dexamethasone-treated cells compared to untreated cells. Upon sequencing and alignment, this segment was identified as belonging to the fifth intron of the Itk gene (Figure 1A). Because this segment was not amplified in non-transcribed samples, we concluded that the segment was derived from an RNA sequence that was retrotranscribed into cDNA.
However, NCBI databases do not contain EST sequences and our attempts to clone a full-length cDNA containing an open reading frame by rapid amplification of cDNA ends (RACE) were unsuccessful. The only PCR product that we obtained was a longer segment belonging to intron 5 of Itk. The reason why an mRNA from an intron was transcribed by reverse transcriptase and amplified by differential display, PCR, and RACE remains unclear and is under investigation. One possibility is that this region has regulatory functions or results from pre-mRNA splicing, which is highly up-regulated by glucocorticoids.

We reasoned that if transcription of the intron is upregulated, Itk transcription should also be up-regulated. Indeed, RNAse protection assay (RPA) confirmed our hypothesis because Itk transcription was up-regulated 4-fold in 3DO cells (Figure 1B). Considering that Itk is a kinase involved in TCR signaling, we investigated the effect of dexamethasone on the expression of other kinases activated by TCR stimulation, focusing our attention on Txk, the other relevant Tec kinase in T cells, and on Lck. In 3DO cells, expression of Lck and Txk was down-regulated by dexamethasone (Figure 1B), thereby suggesting that Itk, Txk, and Lck may play different roles in glucocorticoid-treated lymphocytes.

\section{Dexamethasone in vitro treatment up-regulates the expression of Itk and $T x k$, but not $L c k$, in thymocytes and peripheral T lymphocytes}

Next, we examined the effect of dexamethasone on Itk, $L c k$, and $T x k$ gene expression in murine thymocytes, splenocytes, and lymphocytes isolated from lymph nodes. For this, we used RPA because of its high sensitivity and accuracy in measuring mRNA levels [26,27]. For each probe, the cpm of the fragment protected by the gene under investigation was normalized to that protected by $\beta$-actin. Gene modulation was then quantified as the ratio of gene expression in treated versus untreated samples (Figure 2). Our data show that Itk expression increased in both thymocytes and peripheral T cells (Figure 3A), confirming the data obtained from dexamethasone-treated 3DO cells. Surprisingly, Txk expression also increased, albeit to a lower level than that of Itk (Figure 3B). Although dexamethasone significantly decreased $L c k$ expression in thymocytes as previously demonstrated [28], little to no effect was observed in mature T-cell populations (Figure 3C).

Lck is expressed not only in T cells but also in NK cells, B cells, and dendritic cells (DCs) [28]. The modulation observed in spleen and lymph nodes may derive from modulation of Lck in both $\mathrm{T}$ and other cells. Itk and Txk are expressed almost exclusively in T and NK cells [12] but modulation observed in splenocytes and lymph node $\mathrm{T}$ cells may be due to indirect effects, such as interaction of T cells with other glucocorticoid-treated cells present in the 

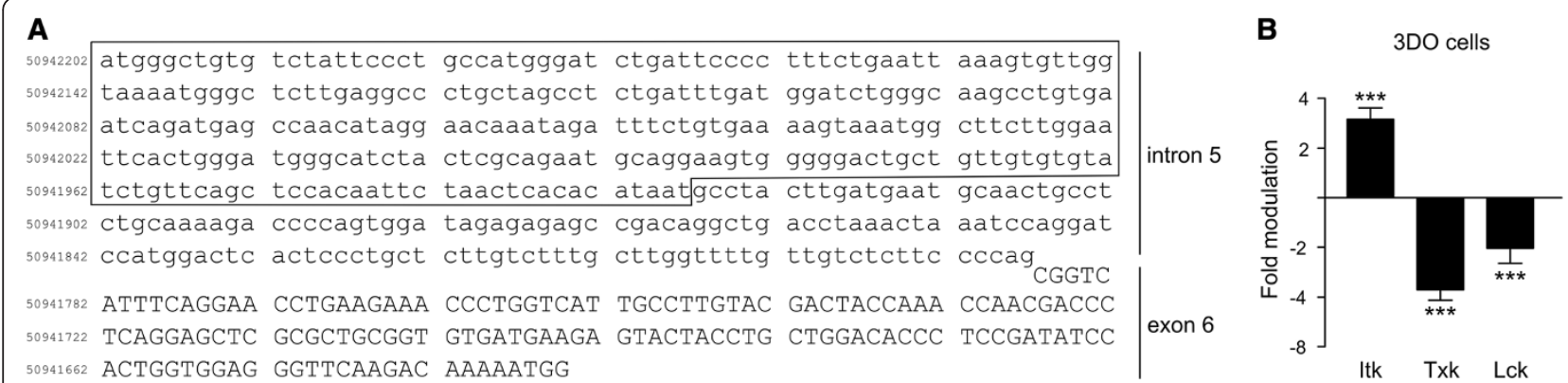

Figure 1 ltk, Txk, and Lck gene expression is modulated by dexamethasone in the T cell hybridoma 3DO. (A) The DNA amplified and isolated by the differential display technique (included in the box) is a 275-bp fragment belonging to intron 5 of the ltk gene in proximity to exon 6. In the original fragment, the first and last $10 \mathrm{bp}$ were identical/complimentary to the OPA (the primer used in differential display) used in the PCR reaction and overlapped only partially with the sequence of intron 5 (data not shown). (B) Itk, Txk, and Lck expression in 3DO cells is modulated by $24-h$ treatment with dexamethasone $(100 \mathrm{nM})$ as demonstrated by the RNAse protection assay. Fold modulation was calculated by dividing the Itk/ $\beta$-actin cpm ratio of DEX-treated cells by that of the respective untreated control. Values represent the mean $( \pm S D)$ of three independent experiments. ${ }^{* *} \mathrm{P}<0.001$, according to Student's t-test comparing the levels of kinase mRNA in dexamethasone-treated cells to those in medium-treated control cells.

culture (e.g., B cells). To confirm that the observed modulation was induced by dexamethasone treatment of $\mathrm{T}$ cells, we purified total, $\mathrm{CD} 4^{+}$and $\mathrm{CD} 8^{+} \mathrm{T}$ cells and evaluated the effect of glucocorticoid treatment by real-time PCR. The results confirmed that $I t k$ and $T x k$ expression in $\mathrm{T}$ cells increases over time similar to expression in lymph node cells from the same animals although the levels were lower (Figure 3D-E). Lck expression was not modulated in either $\mathrm{T}$ cells or lymph node cells (Figure 3F).

\section{Itk, Txk, and Lck are also modulated by dexamethasone treatment in vivo}

We then proceeded to investigate whether dexamethasone affects the expression of these kinases in thymocytes and splenocytes following in vivo treatment. Mice were treated with either two doses of dexamethasone or with saline solution and then sacrificed after 3 or $6 \mathrm{~h}$. The results show that Itk expression is significantly increased upon administration of both low and high doses of dexamethasone and that the increase is dose dependent (Figure 4). Although Itk expression in the thymus increased up to $6 \mathrm{~h}$ after administration, Itk expression in the spleen peaked at $3 \mathrm{~h}$ before returning to control levels after $6 \mathrm{~h}$. Interestingly, despite possible differences between in vitro and in vivo treatments, including cellular redistribution, cross-talk, and pharmacokinetics, treatment with dexamethasone for $3 \mathrm{~h}$ modulated Itk expression similarly in vitro and in vivo. Of note, a slight but significant increase in Itk expression was also observed following saline treatment in both thymocytes and splenocytes (Figure 4A). This increase was most likely due to endogenous production of glucocorticoids following manipulation stress, supporting the idea that Itk modulation can occur with physiologic alteration of endogenous glucocorticoids. The observed up-regulation of Itk mRNA led to increased Itk protein levels as demonstrated by western blot analysis (Figure 4C).

Evaluation of glucocorticoid-induced in vivo modulation of the $T x k$ gene revealed that, compared to saline-treated mice, gene expression was increased slightly in peripheral $\mathrm{T}$ cells after a $3 \mathrm{~h}$ treatment with the highest dexamethasone dose (Figure 5A, right panel), consistent with the reduced responsiveness to dexamethasone treatment observed in vitro. Both low and high dexamethasone doses were effective in promoting $T x k$ modulation after treatment for $6 \mathrm{~h}$. On the contrary, Txk expression did not change significantly in thymocytes following in vivo dexamethasone treatment (Figure 5A, left panel). Compared to saline-treated control animals, dexamethasone-treated mice exhibited strong down-regulation of $L c k$ expression in the thymus (Figure 5B, left panel). This downregulation was dose-dependent and consistent with results from the in vitro experiments. Comparison of salinetreated and untreated mice revealed significantly decreased $L c k$ expression in saline-treated mice (1.7 fold, P $<0.01$, data not shown). However, Lck was up-regulated significantly in mature lymphocytes just $6 \mathrm{~h}$ after administration of the highest dexamethasone dose (Figure 5B, right panel). Taken together, $I t k, T x k$, and $L c k$ were modulated by dexamethasone treatment. Although these kinases are activated upon T-cell activation and participate in overlapping signaling pathways, dexamethasone modulated each kinase differently (summarized in Table 1).

\section{Thymocytes from $\mathrm{Itk}^{-/-}$mice were more sensitive to dexamethasone treatment}

To verify whether dexamethasone-induced Itk up-regu lation plays a functional role, we treated $I t k^{-/-}$thymocytes with dexamethasone and evaluated apoptosis by flow 


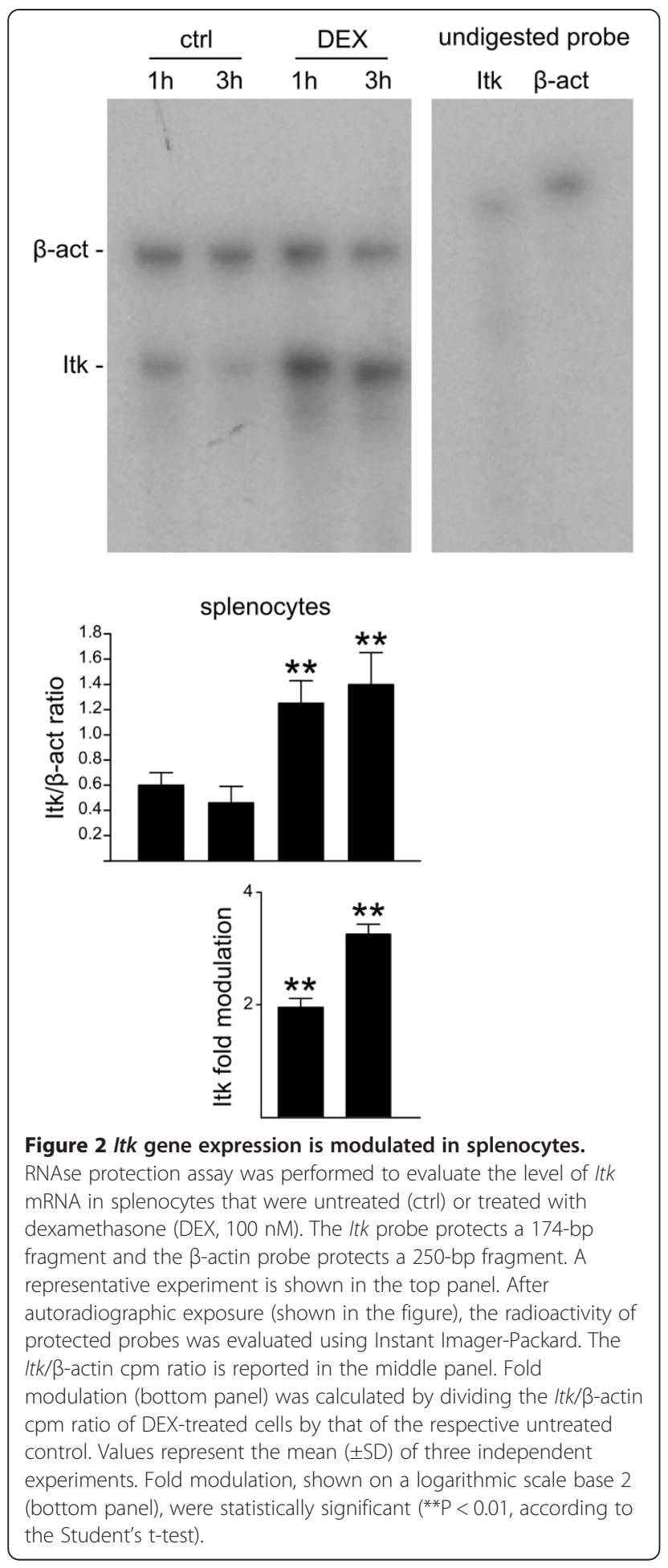

cytometric analysis of propidium iodide-stained cells. Figure 6A and 6B (left panel) show that $I t k^{-/-}$thymocytes were more sensitive to dexamethasone-induced apoptosis than wild type thymocytes. A significantly higher percentage of apoptotic cells was observed even at the lowest dexamethasone concentration. At the same dexamethasone concentration $\left(2.5 \times 10^{-8} \mathrm{M}\right)$, wild type thymocytes exhibited similar levels of apoptosis to that of untreated cells.

Interpretation of this data is complicated by the higher level of spontaneous apoptosis observed in $\mathrm{Itk}^{-/-}$thymocytes compared to wild type thymocytes. To compare the levels of dexamethasone-induced apoptosis, we normalized the data against the levels of spontaneous apoptosis detected and then calculated dexamethasone-specific apoptosis (see Methods section for details). The differences in specific apoptosis between $\mathrm{Itk}^{-/-}$and wild type thymocytes were significant at each tested concentration (Figure 6B).

To evaluate whether this increased dexamethasonespecific apoptosis in $I t k^{-/-}$thymocytes was caused by a greater susceptibility to apoptosis, we evaluated the effect of other pro-apoptotic stimuli. Our data demonstrate that $I t k^{-/-}$thymocytes were not more sensitive to apoptosis induced by anti-CD3 and heath shock (Figure 6C and 6D). Therefore, increased apoptosis in Itk ${ }^{-/-}$thymocytes suggests that Itk plays a protective role in dexamethasone-induced apoptosis, which works to counteract this biological phenomenon.

\section{Discussion}

In several cell types, including $\mathrm{T}$ cells, glucocorticoids modulate the transcription of hundreds of genes whose differential expression changes the fate and function of cells, causing apoptosis, maturation, and differentiation [1-3]. Here we demonstrate that dexamethasone modulates Lck and Tec kinases rapidly in thymocytes and/or mature lymphocytes. If we consider the pro-apoptotic role of dexamethasone in thymocytes, we would expect that genes with a pro-survival and activating function are down-regulated. Surprisingly, while Lck expression was found to be down-regulated, Tec kinase expression was up-regulated by dexamethasone. The regulation of Lck and Tec kinases was not coordinated even in mature lymphocytes, further suggesting that the effects of glucocorticoids in $\mathrm{T}$ cells, and specifically $\mathrm{T}$-cell activation, are complex.

Our study clearly demonstrates for the first time that dexamethasone up-regulates $I t k$ expression in thymocytes. In our previous study, we found that Itk was slightly upregulated in $\mathrm{CD} 4^{+} \mathrm{CD} 8^{+}$double positive thymocytes treated with dexamethasone for $3 \mathrm{~h}$ [23]. However, the upregulation was not validated using other techniques and the increased expression appeared to be low (1.5-fold). The higher level of up-regulation observed in this study (i.e., greater than 2-fold) may be the result of different techniques and/or the presence of $\mathrm{CD}^{+}$and $\mathrm{CD} 8^{+}$singlepositive thymocytes. The up-regulation was very rapid because a greater than 2-fold increase was observed after $1 \mathrm{~h}$ of dexamethasone treatment in vitro. Furthermore, these data are quantitatively relevant because an approximately 


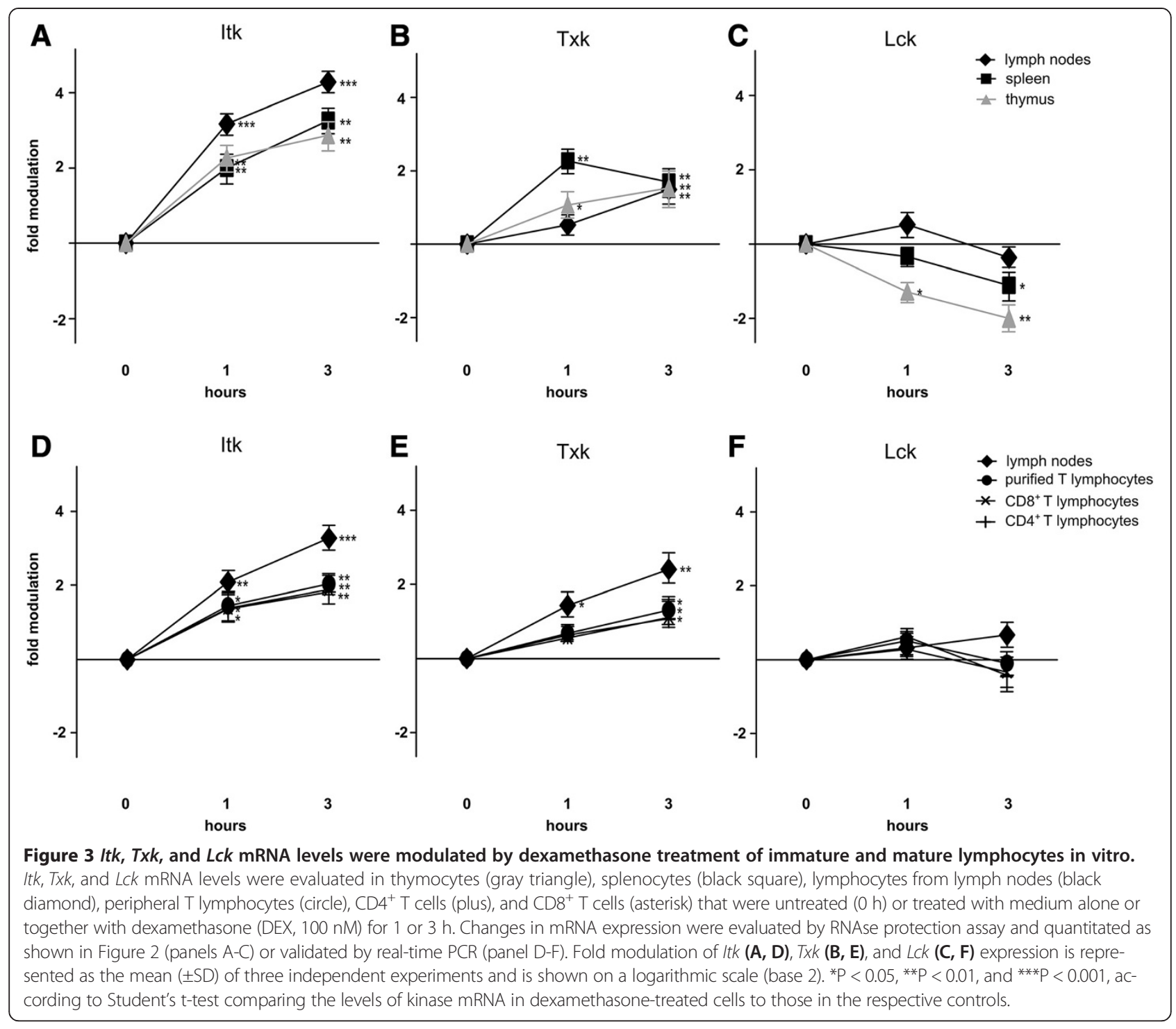

4-fold increase was observed after treatment for $6 \mathrm{~h}$ in vivo, where the tested concentration was about 10fold higher than that tested in vitro. The up-regulation of $I t k$ in thymocytes following in vivo treatment confirms the relevance of the in vitro data and demonstrates that it is present despite the thymic microenvironment, including TCR triggering and endogenous glucocorticoids. Itk upregulation protects thymocytes from dexamethasoneinduced apoptosis, as demonstrated by the specific increased sensitivity of $\mathrm{Itk}^{-/-}$thymocytes to glucocorticoids along with their decreased/unchanged sensitivity to other pro-apoptotic stimuli. In this context, the increased level of $I t k$ expression observed after in vivo treatment with a high dose of dexamethasone may be due, at least in part, to protection from dexamethasoneinduced apoptosis for those thymocytes with high levels of $I t k$ expression.
A protective function for up-regulation of prosurvival genes in cells undergoing apoptosis is well supported by the literature. For example, a previous study from our group demonstrated that 3-h dexamethasone treatment of $\mathrm{CD}_{4}^{+} \mathrm{CD}^{+}$double-positive thymocytes resulted in transcriptional regulation of at least 10 genes with known protective functions [23], including interleukin-7 receptor, which delivers anti-apoptotic and activating signals to cells upon activation, and FKBP5 immunophilin, which inhibits the interaction between glucocorticoids and their receptor. Interleukin-7 receptor was also found to be up-regulated by glucocorticoids in mature $\mathrm{T}$ cells [29]. Moreover, FKBP5 has been reported to be upregulated when endogenous glucocorticoid overproduction is stimulated [30].

Analysis of the presence of glucocorticoid responsive elements (GRE) revealed a very well-conserved GRE 

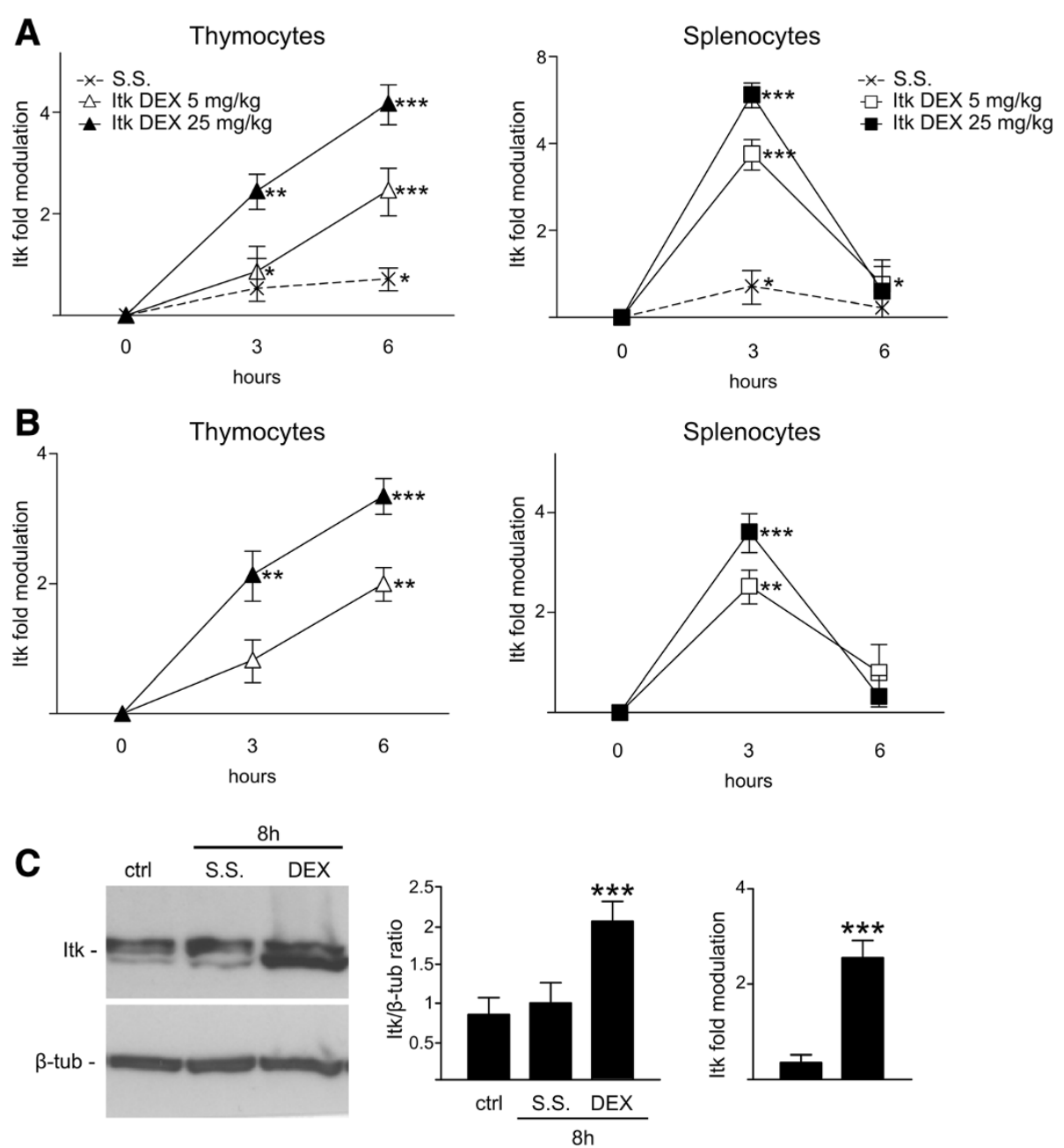

Figure 4 Itk expression is modulated by dexamethasone treatment in thymocytes and splenocytes in vivo. The level of Itk mRNA was evaluated in thymocytes (left panels) and splenocytes (right panels) isolated from mice treated with $5 \mathrm{mg} / \mathrm{kg}$ (empty symbol) or $25 \mathrm{mg} / \mathrm{kg}$ (black symbol) dexamethasone (DEX), or saline solution (S.S., asterisk). The animals were sacrificed 3 or $6 \mathrm{~h}$ after treatment. Changes in mRNA level were evaluated by RNA protection assay and evaluated as shown in Figure 2. Fold modulation is represented as the mean ( \pm SD) of three independent experiments, with each experiment performed on cells pooled from three animals. The data are graphed on a logarithmic scale (base 2). ${ }^{*} \mathrm{P}<0.05$, ${ }^{*} \mathrm{P}<0.01$, and ${ }^{* * *} \mathrm{P}<0.001$, according to Student's t-test comparing the levels of Itk mRNA in cells from treated animals to that in cells from untreated animals (A) or from saline-treated animals (B). (C) Levels of Itk protein were evaluated in thymocytes from mice treated for $8 \mathrm{~h}$ with $5 \mathrm{mg} / \mathrm{kg}$ dexamethasone (DEX) or saline solution (S.S.). The left panel shows one representative experiment $(\mathrm{n}=4)$. After densitometric analysis, the Itk/ $\beta$-tubulin ratio (middle panel) and fold modulation (right panel) were calculated. ${ }^{* *} \mathrm{P}<0.001$, according to Student's t-test comparing the level of Itk protein in thymocytes from DEX-treated animals to that in thymocytes from untreated and saline-treated animals.

motif within intron 9 of the $I t k$ gene (Additional file 1 : Table S1 and Additional file 2: Figure S1) that may account for $I t k$ up-regulation in thymocytes and peripheral $\mathrm{T}$ cells. Surprisingly, we also found a well-conserved negative GRE (nGRE) within the promoter region of $I t k$. Differences in the extent of $I t k$ up-regulation and its kinetics in cell lines, thymocytes, splenocytes, lymphocytes from lymph nodes, and purified $\mathrm{T}$ lymphocytes may be due to the balanced effects of GRE and nGRE, transcription factors present in each cell type, basal level of expression of Itk in different cell types, and other signals modulating the effects of glucocorticoids. Interestingly,
Itk up-regulation is higher in lymphocytes from lymph nodes than in splenocytes and $\mathrm{T}$ cells from splenocytes, suggesting that the microenvironment from which $\mathrm{T}$ cells are derived influences the effects of glucocorticoids.

Our data also reveal that $L c k$ is down-regulated by dexamethasone in thymocytes. This effect was observed as early as $1 \mathrm{~h}$ after in vitro treatment and continued up to $6 \mathrm{~h}$ in vivo. To the best of our knowledge, Lck downregulation has been reported by other studies but only following long treatment times. In rat thymocytes, a 24$\mathrm{h}$ glucocorticoid treatment decreased Lck protein level [31]. Moreover, in murine thymocytes (from a different 

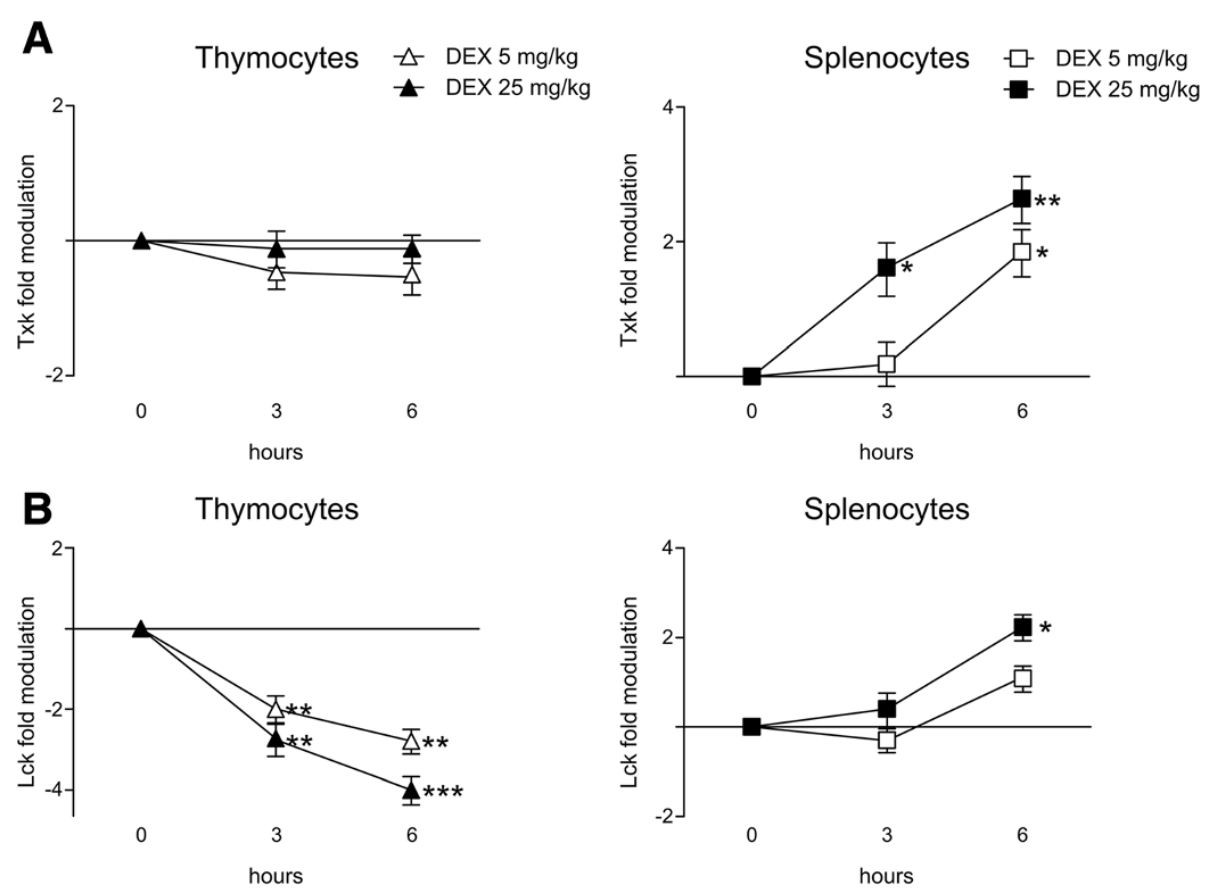

Figure 5 Txk and Lck gene expression is modulated by dexamethasone treatment of thymocytes and splenocytes in vivo. Levels of Txk (A) and $L c k$ (B) mRNA were evaluated in thymocytes (left panel) and splenocytes (right panel) from mice treated with $5 \mathrm{mg} / \mathrm{kg}$ (empty symbol) or $25 \mathrm{mg} / \mathrm{kg}$ (black symbol) dexamethasone, or saline solution. The animals were sacrificed 3 or $6 \mathrm{~h}$ after treatment. Changes in mRNA level were evaluated by RNAse protection assay as shown in Figure 2. Fold modulation is represented as the mean ( \pm SD) of three independent experiments, with each experiment performed on cells pooled from three animals. The data are graphed on a logarithmic scale (base 2 ). ${ }^{*} P<0.05$, ${ }^{*} \mathrm{P}<0.01$, and ${ }^{* *} \mathrm{P}<0.001$, according to Student's t-test comparing the levels of kinase mRNA in cells from treated animals to that in cells from saline-treated animals.

strain to the one used here), decreased Lck mRNA expression was observed after $12-24 \mathrm{~h}$ of glucocorticoid treatment [28]. Inhibition of this kinase by treatment with shRNA and the Src inhibitor dasatinib enhanced thymocyte sensitivity to dexamethasone [28], suggesting that Lck protects cells from glucocorticoid-induced apoptosis. Low levels of Lck were found in T cells from the spleen and lymph nodes of mice suffering from an experimental graft-versus-host reaction (GVHR), and Lck down-regulation was demonstrated to be dependent on glucocorticoids [32]. In the same model, the level of
Lck in thymocytes remained unchanged with respect to the control. Taken together, our results (short-term treatment) are consistent with these reports (long-term treatment), and suggest that glucocorticoid-mediated $L c k$ modulation is complex and time- and concentrationdependent.

Surprisingly, we did not find sequences with a match to nGRE matrixes greater than 0.85 (i.e., exhibiting a high probability of binding GR and inhibiting transcription) within the Lck promoter region. Instead, we found a sequence with a match to the GRE matrixes greater than 0.85

Table 1 Summary of kinase modulation following dexamethasone treatment

\begin{tabular}{|c|c|c|c|c|}
\hline & & \multicolumn{3}{|c|}{ Effect of dexamethasone treatment } \\
\hline & & In vitro $3 \mathrm{~h}$ treatment & In vivo $3 \mathrm{~h}$ treatment $(25 \mathrm{mg} / \mathrm{kg})$ & In vivo $6 \mathrm{~h}$ treatment $(25 \mathrm{mg} / \mathrm{kg})$ \\
\hline \multirow[t]{3}{*}{ Thymocytes } & Itk & $\uparrow \uparrow \uparrow$ & $\uparrow \uparrow$ & $\uparrow \uparrow \uparrow$ \\
\hline & Txk & $\uparrow$ & $=$ & $=$ \\
\hline & Lck & $\downarrow \downarrow$ & $\downarrow \downarrow \downarrow$ & $\downarrow \downarrow \downarrow$ \\
\hline \multirow[t]{3}{*}{ Lymphocytes from peripheral organs } & ltk & $\uparrow \uparrow \uparrow$ & $\uparrow \uparrow \uparrow$ & $=$ \\
\hline & $T x k$ & $\uparrow \uparrow$ & $\uparrow \uparrow$ & $\uparrow \uparrow \uparrow$ \\
\hline & Lck & $=$ & $=$ & $\uparrow \uparrow$ \\
\hline
\end{tabular}

$=$,gene expression difference between treated and medium-treated samples/animals is not significant;

$\uparrow$ or $\downarrow$,The difference between treated and untreated samples is significant and has a fold modulation (increase or decrease) lower than 1.6 or higher than -1.6.

$\uparrow \uparrow$ or $\downarrow \downarrow$,The difference between treated and untreated samples is significant and has a fold modulation comprised between 1.6 and 2.2 or -1.6 and -2.2 .

$\uparrow \uparrow \uparrow$ or $\downarrow \downarrow \downarrow$,The difference between treated and untreated samples is significant and has a fold modulation higher than 2.2 or lower than -2.2 . 

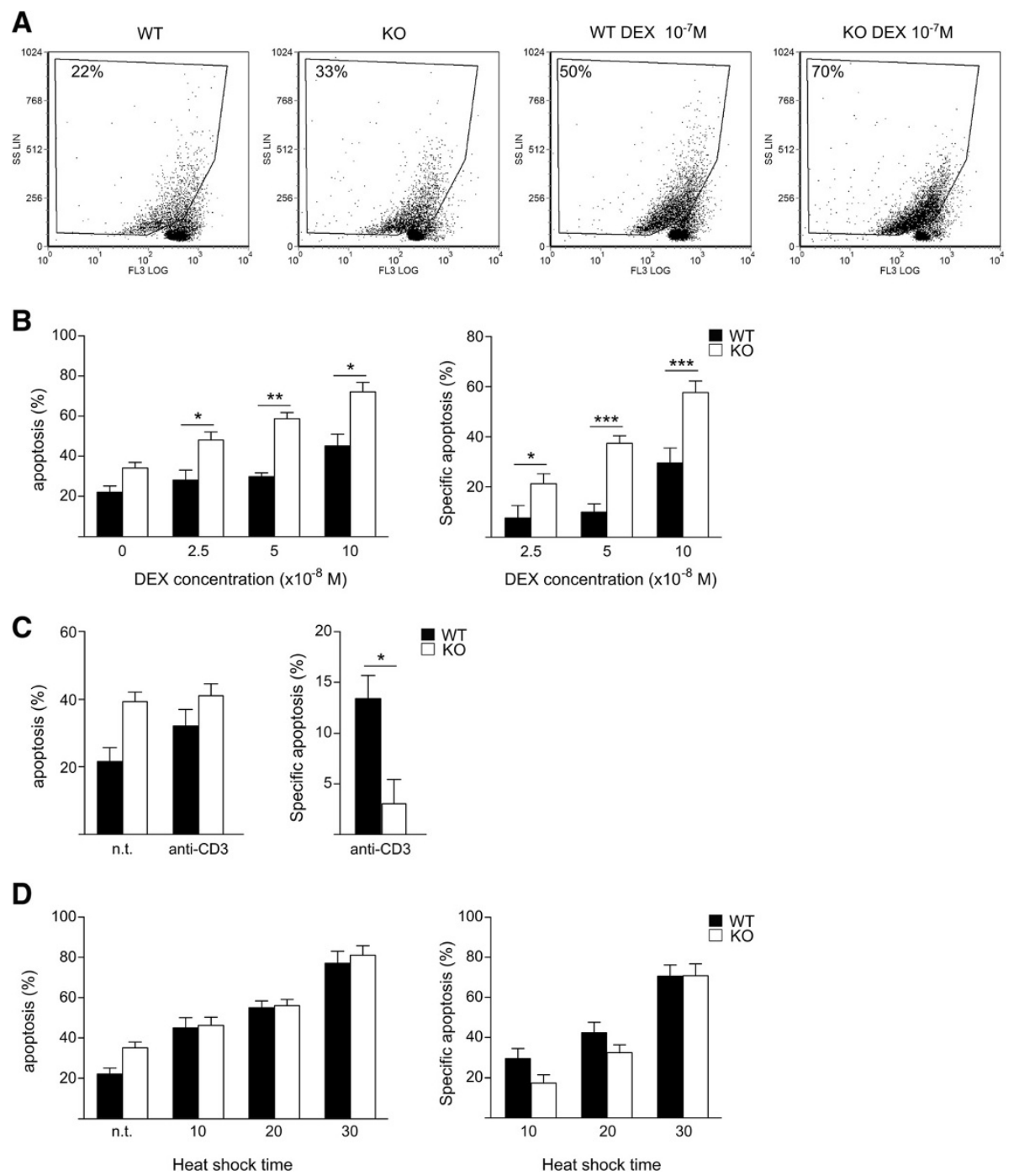

Figure $6 \mathrm{ltk}^{-/-}$thymocytes are more sensitive than wild type thymocytes to apoptosis induced by dexamethasone but not by TCR activation or heat shock. (A) $1 \mathrm{tk}^{-/-}$(KO) and wild type (WT) thymocytes were left untreated or treated with $10^{-7} \mathrm{M}$ dexamethasone (DEX) for $18 \mathrm{~h}$ before staining with a hypotonic solution of propidium iodide followed by flow cytometric analysis for apoptotic cells. One representative experiment performed on cells pooled from four mice is shown. The percentage of apoptotic cells within the gate is reported. (B) The percentage of apoptotic cells following treatment with the specified concentration of DEX is represented as the mean $( \pm$ SD) of four independent experiments, performed on cells pooled from three to four mice. Because the level of spontaneous apoptosis was higher in KO than WT thymocytes, apoptosis in DEX-treated cells was normalized using the respective level of spontaneous apoptosis and the DEX-specific apoptosis was calculated as specified in Methods (right panel). (C) KO and WT thymocytes were treated with anti-CD3 antibody for $18 \mathrm{~h}$ and apoptosis was evaluated as above. The percentage of apoptotic cells is represented as the mean $( \pm \mathrm{SD}$ ) of four independent experiments, with each experiment performed on cells pooled from three or four mice. Specific apoptosis is shown in the right panel. (D) KO and WT thymocytes were incubated at $43^{\circ} \mathrm{C}$ for the specified times and apoptosis was evaluated after a further $18 \mathrm{~h}$ as described above. The percentage of apoptotic cells is represented as the mean $( \pm \mathrm{SD})$ of four independent experiments, with each experiment performed on cells pooled from three or four mice. Specific apoptosis is shown in the right panel. ${ }^{*} \mathrm{P}<0.05$, ${ }^{*} \mathrm{P}<0.01$, and ${ }^{* *} \mathrm{P}<0.001$, according to Student's t-test comparing KO with WT apoptosis (left panel) or DEX-specific apoptosis (right panel).

(Additional file 1: Table S1 and Additional file 2: Figure S1). Because data from this and other studies show that glucocorticoids down-regulate Lck expression in thymocytes, we can conclude that this effect is due to heterodimerization of activated GR to transcription factors and that the GRE seems to be inactive in these cells. On the contrary, the up- regulation found in splenocytes after treatment for $6 \mathrm{~h}$ in vivo may be due to the GRE.

Investigation of the $T x k$ promoter revealed three and two very well-conserved GRE and nGRE motifs, respectively (Additional file 1: Table S1 and Additional file 2: Figure S1). These data suggest that $T x k$ expression is strictly controlled 
by glucocorticoids and that differences in glucocorticoiddependent Txk modulation between thymocytes and $\mathrm{T}$ lymphocytes from peripheral organs may reflect the balance between GRE- and nGRE-mediated regulation.

Glucocorticoids affect peripheral immune responses by inhibiting $\mathrm{T}$ cell immunity at several stages of the activation cascade. Because the thymic epithelium produces steroids, it is reasonable to hypothesize that endogenous glucocorticoids also play a role in controlling T-cell development [33,34]. Brewer and colleagues showed that apoptosis induced by TCR triggering is mediated by glucocorticoids in murine thymocytes. Reports have also described glucocorticoidmediated transcriptional regulation of TCR complex proteins $[21,35,36]$, as well as modulation of TCR function and signaling transduction [37-40]. In particular, glucocorticoid treatment has been shown to modulate kinase activity in activated $\mathrm{T}$ cells [41]. As both peripheral responsiveness and thymic differentiation appear to be regulated by the quantity and quality of intracellular signals triggered by TCR activation, the interference of glucocorticoids on the expression of kinases downstream of the TCR may contribute to the effect of endogenous and pharmacological glucocorticoids on T cells.

Our study demonstrates for the first time that both Tec kinases are up-regulated in splenocytes and lymphocytes. However, Itk up-regulation was more rapid and quantitatively relevant considering that the basal levels of Itk expression are higher than that of $T x k$ in mature lymphocytes [12]. Several findings suggest that Itk activity favors differentiation towards the Th2 phenotype [14-16,42]. The various effects of glucocorticoids on $\mathrm{T}$ cell differentiation in the different experimental models and human diseases depend on several factors; however, data suggest that glucocorticoids favor Th2 and Treg differentiation of $\mathrm{CD}^{+} \mathrm{T}$ cells $[5,7,8]$. In this context, it is likely that dexamethasone-induced Itk upregulation is at least partly responsible for the Th2-polarizing effects of glucocorticoids. Moreover, Itk seems to favor Th17-induced $\mathrm{T}$ regulatory cell (iTreg) polarization [43] whereas glucocorticoids enhance the Th17/Th1 imbalance in patients with systemic lupus erythematosus [44].

\section{Conclusions}

Modulation of TCR signaling by glucocorticoids is known to involve several mechanisms, including regulation of TCR complex subunit expression and kinase activity. In this study, we show that $I t k$, $T x k$, and $L c k$ expression is modulated by dexamethasone in thymocytes and mature lymphocytes, demonstrating another mechanism by which glucocorticoids modulate T-cell activation. Furthermore, we reveal that dexamethasone-driven Itk up-regulation plays a protective role in glucocorticoid-induced thymocyte apoptosis. Dexamethasone was also found to induce differential expression of $I t k$ and $T x k$ in mature lymphocytes, possibly favoring Tcell polarization. Future studies are required to address the actual in vivo relevance of such a mechanism.

\section{Additional files}

Additional file 1: Table S1. Putative glucocorticoid responsive element (GRE) and negative GRE (nGRE) sites within the murine Itk, Txk and Lck genes.

Additional file 2: Figures S1. The putative Glucocorticoid Responsive Element (GRE) and negative GRE (nGRE) sites in murine Itk, Txk and Lck genes.

\section{Abbreviations}

Ab: Antibody; Itk: IL2 inducible T cell kinase; Itk ${ }^{-1-}$ : Itk knock-out; Lck: Lymphocyte protein tyrosine kinase; RACE: Rapid amplification of cDNA ends; RPA: RNAse protection assay; TCR: T-cell receptor; Th: T helper; Txk: Txk tyrosine kinase; $\mathrm{Txk}^{-1-}$ : Txk knock-out.

\section{Competing interests}

The authors declare that they have no competing interest.

\section{Authors' contributions}

GMP conducted PCR, RPA, and in vivo experiments, prepared figures, and contributed to the writing of the manuscript; KF conducted PCR, RPA, western blotting, apoptosis, and in vivo experiments; PM participated in research design, conducted PCR experiments, and performed data analysis; SR conducted RPA and in vivo experiments; LC conducted real-time PCR experiments; GM participated in research design and contributed to the writing of the manuscript; EM conducted in vivo experiments and contributed to the writing of the manuscript; OB conducted RPA and western blotting experiments; SB performed in silico analysis of promoters and conducted apoptosis and in vivo experiments; GN participated in research design, performed data analysis, and wrote the manuscript; CR participated in research design and contributed to the writing of the manuscript. All authors read and approved the final manuscript.

\section{Acknowledgments}

This work was supported by Cassa di Risparmio di Perugia.

\section{Author details}

${ }^{1}$ Department of Medicine, University of Perugia, Perugia, Italy. ${ }^{2}$ Department of Experimental Medicine, University of Perugia, Perugia, Italy. ${ }^{3}$ Department of Pharmacology, Faculty of Medicine, Catholic University of the Sacred Heart, Rome, Italy. ${ }^{4}$ Istituto di Ricovero e Cura a Carattere Scientifico (IRCCS) Centro Neurolesi "Bonino-Pulejo", Messina, Italy. ${ }^{5}$ Department of Medicine, Section of Pharmacology, Severi Square 1, University of Perugia, I-06132 San Sisto, Perugia, Italy.

Received: 11 February 2014 Accepted: 25 June 2014 Published: 3 July 2014

\section{References}

1. Busillo JM, Cidlowski JA: The five Rs of glucocorticoid action during inflammation: ready, reinforce, repress, resolve, and restore. Trends Endocrinol Metab 2013, 24:109-119.

2. Vandevyver $S$, Dejager $L$, Tuckermann J, Libert C: New insights into the anti-inflammatory mechanisms of glucocorticoids: an emerging role for glucocorticoid-receptor-mediated transactivation. Endocrinology 2013, 154:993-1007.

3. Ronchetti S, Migliorati G, Riccardi C: Glucocorticoid-induced immunomodulation. In Molecular Immunotoxicology. Firstth edition. Edited by Van Loveren H, Corsini E. Wiley-VCH Verlag Gmbh \& Co. KGaA; 2015. in press.

4. Wiegers GJ, Labeur MS, Stec IE, Klinkert WE, Holsboer F, Reul JM: Glucocorticoids accelerate anti-T cell receptor-induced T cell growth. J Immunol 1995, 155:1893-1902.

5. Ramirez F, Fowell DJ, Puklavec M, Simmonds S, Mason D: Glucocorticoids promote a TH2 cytokine response by CD4+ T cells in vitro. J Immunol 1996, 156:2406-2412.

6. Cannarile L, Fallarino F, Agostini M, Cuzzocrea S, Mazzon E, Vacca C, Genovese T, Migliorati G, Ayroldi E, Riccardi C: Increased GILZ expression in transgenic mice up-regulates Th-2 lymphokines. Blood 2006, 107:1039-1047. 
7. Bereshchenko O, Coppo M, Bruscoli S, Biagioli M, Cimino M, Frammartino T, Sorcini D, Venanzi A, Di Sante M, Riccardi C: GILZ Promotes Production of Peripherally Induced Treg Cells and Mediates the Crosstalk between Glucocorticoids and TGF-beta Signaling. Cell Rep 2014, 7:464-475.

8. Liberman AC, Druker J, Refojo D, Holsboer F, Arzt E: Glucocorticoids inhibit GATA-3 phosphorylation and activity in T cells. FASEB J 2009, 23:1558-1571

9. Molina TJ, Kishihara K, Siderovski DP, van Ewijk W, Narendran A, Timms E, Wakeham A, Paige CJ, Hartmann KU, Veillette A, Davidson D, Mak TW: Profound block in thymocyte development in mice lacking p56lck. Nature 1992, 357:161-164.

10. Smith-Garvin JE, Koretzky GA, Jordan MS: T cell activation. Annu Rev Immunol 2009, 27:591-619.

11. Sahu N, August A: ITK inhibitors in inflammation and immune-mediated disorders. Curr Top Med Chem 2009, 9:690-703.

12. Gomez-Rodriguez J, Kraus ZJ, Schwartzberg PL: Tec family kinases Itk and Rlk / Txk in T lymphocytes: cross-regulation of cytokine production and T-cell fates. FEBS J 2011, 278:1980-1989.

13. Schaeffer EM, Debnath J, Yap G, McVicar D, Liao XC, Littman DR, Sher A, Varmus HE, Lenardo MJ, Schwartzberg PL: Requirement for Tec kinases Rlk and Itk in T cell receptor signaling and immunity. Science 1999, 284:638-641.

14. Mueller $C$, August A: Attenuation of immunological symptoms of allergic asthma in mice lacking the tyrosine kinase ITK. J Immunol 2003, 170:5056-5063.

15. Fowell DJ, Shinkai K, Liao XC, Beebe AM, Coffman RL, Littman DR, Locksley RM: Impaired NFATc translocation and failure of Th2 development in Itk-deficient CD4+ T cells. Immunity 1999, 11:399-409.

16. Schaeffer EM, Yap GS, Lewis CM, Czar MJ, McVicar DW, Cheever AW, Sher A, Schwartzberg PL: Mutation of Tec family kinases alters T helper cell differentiation. Nat Immunol 2001, 2:1183-1188.

17. Suzuki N, Nara K, Suzuki T: Skewed Th1 responses caused by excessive expression of Txk, a member of the Tec family of tyrosine kinases, in patients with Behcet's disease. Clin Med Res 2006, 4:147-151.

18. Shimonkevitz R, Kappler J, Marrack P, Grey H: Antigen recognition by H-2-restricted T cells. I. Cell-free antigen processing. J Exp Med 1983, 158:303-316.

19. Liang P, Pardee AB: Differential display of mRNA by PCR. In Current Protocols in Molecular Biology. 25th edition. Edited by Ausubel FM, Brent R, Kingston RE, Moore DD, Seidman JG, Smith JA, Strhul K. 2001. Unit 25B 23.

20. Schaefer $B C$ : Revolutions in rapid amplification of cDNA ends: new strategies for polymerase chain reaction cloning of full-length cDNA ends. Anal Biochem 1995, 227:255-273.

21. Ronchetti S, Nocentini G, Giunchi L, Bartoli A, Moraca R, Riccardi C, Migliorati G: Short-term dexamethasone treatment modulates the expression of the murine TCR zeta gene locus. Cell Immunol 1997, 178:124-131.

22. Spinicelli S, Nocentini G, Ronchetti S, Krausz LT, Bianchini R, Riccardi C: GITR interacts with the pro-apoptotic protein Siva and induces apoptosis. Cell Death Differ 2002, 9:1382-1384

23. Bianchini R, Nocentini G, Krausz LT, Fettucciari K, Coaccioli S, Ronchetti S, Riccardi C: Modulation of pro- and antiapoptotic molecules in doublepositive (CD4+CD8+) thymocytes following dexamethasone treatment. J Pharmacol Exp Ther 2006, 319:887-897.

24. Ronchetti S, Nocentini G, Bianchini R, Krausz LT, Migliorati G, Riccardi C: GITR lowers the threshold of CD28 costimulation in CD8+ T cells. J Immunol 2007, 179:5916-5926.

25. Riccardi C, Nicoletti I: Analysis of apoptosis by propidium iodide staining and flow cytometry. Nat Protoc 2006, 1:1458-1461.

26. Qu Y, Boutjdir M: RNase protection assay for quantifying gene expression levels. Methods Mol Biol 2007, 366:145-158.

27. Venkatesh LK, Fasina O, Pintel DJ: RNAse mapping and quantitation of RNA isoforms. Methods Mol Biol 2012, 883:121-129.

28. Harr MW, Caimi PF, MCColl KS, Zhong F, Patel SN, Barr PM, Distelhorst CW: Inhibition of Lck enhances glucocorticoid sensitivity and apoptosis in lymphoid cell lines and in chronic lymphocytic leukemia. Cell Death Differ 2010, 17:1381-1391.

29. Franchimont D, Galon J, Vacchio MS, Fan S, Visconti R, Frucht DM, Geenen V, Chrousos GP, Ashwell JD, O'Shea JJ: Positive effects of glucocorticoids on T cell function by up-regulation of IL-7 receptor alpha. J Immunol 2002, $168: 2212-2218$.
30. Dunbar DR, Khaled H, Evans LC, Al-Dujaili EA, Mullins LJ, Mullins JJ, Kenyon CJ, Bailey MA: Transcriptional and physiological responses to chronic ACTH treatment by the mouse kidney. Physio/ Genomics 2010, 40:158-166.

31. Garcia-Welsh A, Laskin DL, Shuler RL, Laskin JD: Cellular depletion of p56lck during thymocyte apoptosis. J Leukoc Biol 1994, 56:528-532.

32. Desbarats J, You-Ten KE, Lapp WS: Levels of p56lck and p59fyn are reduced by a glucocorticoid-dependent mechanism in graft-versus-host reaction-induced T cell anergy. Cell Immunol 1995, 163:10-18.

33. Vacchio MS, Papadopoulos V, Ashwell JD: Steroid production in the thymus: implications for thymocyte selection. J Exp Med 1994, 179:1835-1846.

34. Brewer JA, Kanagawa O, Sleckman BP, Muglia L: Thymocyte apoptosis induced by $\mathrm{T}$ cell activation is mediated by glucocorticoids in vivo. $\mathrm{J}$ Immunol 2002, 169:1837-1843.

35. Migliorati G, Bartoli A, Nocentini G, Ronchetti S, Moraca R, Riccardi C: Effect of dexamethasone on T-cell receptor/CD3 expression. Mol Cell Biochem 1997, 167:135-144.

36. Nambiar MP, Enyedy EJ, Fisher CU, Warke VG, Juang YT, Tsokos GC: Dexamethasone modulates TCR zeta chain expression and antigen receptor-mediated early signaling events in human T lymphocytes. Cell Immunol 2001, 208:62-71.

37. Nambiar MP, Enyedy EJ, Fisher CU, Warke VG, Tsokos GC: High dose of dexamethasone upregulates TCR/CD3-induced calcium response independent of TCR zeta chain expression in human T lymphocytes. J Cell Biochem 2001, 83:401-413.

38. Cifone MG, Migliorati G, Parroni R, Marchetti C, Millimaggi D, Santoni A, Riccardi C: Dexamethasone-induced thymocyte apoptosis: apoptotic signal involves the sequential activation of phosphoinositide-specific phospholipase C, acidic sphingomyelinase, and caspases. Blood 1999, 93:2282-2296.

39. Lowenberg M, Verhaar AP, van den Brink GR, Hommes DW: Glucocorticoid signaling: a nongenomic mechanism for T-cell immunosuppression. Trends Mol Med 2007, 13:158-163.

40. Ayroldi E, Zollo O, Bastianelli A, Marchetti C, Agostini M, Di Virgilio R, Riccardi C: GILZ mediates the antiproliferative activity of glucocorticoids by negative regulation of Ras signaling. J Clin Invest 2007, 117:1605-1615.

41. Van Laethem F, Baus E, Smyth LA, Andris F, Bex F, Urbain J, Kioussis D, Leo O: Glucocorticoids attenuate T cell receptor signaling. J Exp Med 2001, 193:803-814.

42. Miller AT, Wilcox HM, Lai Z, Berg LJ: Signaling through Itk promotes T helper 2 differentiation via negative regulation of T-bet. Immunity 2004 21:67-80.

43. Gomez-Rodriguez J, Sahu N, Handon R, Davidson TS, Anderson SM, Kirby MR, August A, Schwartzberg PL: Differential expression of interleukin-17A and $-17 \mathrm{~F}$ is coupled to $\mathrm{T}$ cell receptor signaling via inducible $\mathrm{T}$ cell kinase. Immunity 2009, 31:587-597.

44. Prado C, de Paz B, Gomez J, Lopez P, Rodriguez-Carrio J, Suarez A: Glucocorticoids enhance Th17/Th1 imbalance and signal transducer and activator of transcription 3 expression in systemic lupus erythematosus patients. Rheumatology (Oxford) 2011, 50:1794-1801.

doi:10.1186/2050-6511-15-35

Cite this article as: Petrillo et al:: Transcriptional regulation of kinases downstream of the T cell receptor: another immunomodulatory mechanism of glucocorticoids. BMC Pharmacology and Toxicology 2014 15:35. 